\title{
Integer Frequency Offset Estimation for OFDM Systems with Residual Timing Offset over Frequency Selective Fading Channels
}

\author{
Danping Li, Yongzhao Li, Member, IEEE, Hailin Zhang, Member, IEEE, \\ Leonard J. Cimini, Jr., Fellow, IEEE, and Yuguang Fang, Fellow, IEEE
}

\begin{abstract}
Accurate integer frequency offset (IFO) estimation is crucial for OFDM systems, especially in the presence of frequency-selective fading and residual timing offset (RTO). For existing algorithms, however, it is still a challenge to obtain a good tradeoff among the estimation performance, complexity and spectrum overhead. In this paper, we propose a novel cross ambiguity function (CAF) based IFO estimator using only one training sequence. By designing the training sequence which has only one single sharp peak on its ambiguity function surface, a highly accurate and full acquisition range estimation of the IFO can be obtained in the presence of frequency-selective fading and RTO. Moreover, the adoption of the CAF expression in terms of time-domain signals ensures the complexity of the proposed algorithm is relatively low. Simulation results verify its superior accuracy in frequency-selective fading channels and in the presence of RTO.
\end{abstract}

Index Terms-cross ambiguity function, integer frequency offset, residual timing offset, frequency-selective fading.

\section{INTRODUCTION}

$\mathbf{O}$ RTHOGONAL frequency division multiplexing (OFD$\mathrm{M})$ is a proven technique for high data rate transmission over frequency-selective fading channels [1]. OFDM, however, is extremely sensitive to the frequency offset. In practice, the normalized frequency offset is divided into an integer part (multiple of the subcarrier spacing) and a fractional part (less than one-half of the subcarrier spacing) so that they can be estimated separately. If not accurately estimated and compensated, the fractional frequency offset (FFO) can destroy the orthogonality of the subcarriers and result in inter-carrier interference [2], while the integer frequency offset (IFO) will lead to a circular shift of the subcarrier indices and degrade performance.

For OFDM systems, frequency offset estimation is usually performed after coarse timing estimation, i.e., the estimation

Copyright (c) 2012 IEEE. Personal use of this material is permitted. However, permission to use this material for any other purposes must be obtained from the IEEE by sending a request to pubs-permissions @ieee.org.

This work was supported by the National Natural Science Foundation of China (61072069), the Important National Science \& Technology Specific Projects (2011ZX03003-001-04, 2012ZX03003012-003), the Fundamental Research Funds for the Central Universities (72001859), the State Key Laboratory of Integrated Services Network (ISN090105) and the 111 Project (B08038).

D. Li, Y. Li and H. Zhang are with the State Key Laboratory of Integrated Services Networks, Xidian University, Xi'an, Shaanxi, 710071, China (e-mail: dpli@mail.xidian.edu.cn). L. Cimini is with the Department of Electrical and Computer Engineering, University of Delaware, DE, USA. Y. Fang is with Department of Electrical and Computer Engineering, University of Florida, FL, USA of the start position of the FFT window. So far, although sophisticated timing synchronization schemes can achieve high accuracy for most applications [3], it is very hard to get a perfect time synchronization in the presence of multipath channel fading and noise [4]. Furthermore, even though the linear phase shift caused by a timing error within the ISI-free region of the cyclic prefix (CP) [3] can be easily recovered by channel equalization, the residual timing offset (RTO) may reduce the accuracy of the IFO and degrade the system. Hence, the robustness to RTO is one of the most important considerations in IFO estimation design.

Typically, IFO estimation that is also valid in the presence of RTO can be carried out via time-domain or frequencydomain correlation-based approaches using dedicated training sequences [3][5]-[7]. In [3], a training sequence with a repetitive structure, consisting of multiple identical parts multiplied by a sign pattern, is adopted. However, the estimation range is limited by the number of identical parts, and the number of identical subparts. To obtain a full acquisition range, Schmidl and Cox proposed to use two consecutive differentially encoded training sequences to estimate IFO [5], which we label $\mathrm{S} \& \mathrm{C}$. A modified version of $\mathrm{S} \& \mathrm{C}$ was proposed in [6] using two training sequences with the same structure. To reduce the training overhead, a differential OFDM (DOFDM) based IFO estimation method using only one training sequence is proposed in [7]. However, the estimation performance could degrade severely if the channel is frequency selective. To solve this problem, a maximum-likelihood (ML) approach for IFO estimation is proposed in [8]; however, an assumption of perfect timing synchronization is made. The IFO estimation performance is very sensitive to the RTO even within the ISIfree range of the CP. To the best of our knowledge, none of the existing approaches can achieve a good performance tradeoff among accuracy, training overhead, complexity and IFO acquisition range in the presence of RTO and frequency selectivity.

Different from these approaches, we investigate IFO estimation from the perspective of the ambiguity function (AF) characteristics of the training sequence. Specifically, we propose a cross ambiguity function (CAF) based IFO estimation algorithm for OFDM systems that has full acquisition range and good robustness to RTO. With the use of the energydetection based metric, high-resolution IFO estimation for frequency-selective fading channels with RTO can be obtained by designing a training sequence having an $\mathrm{AF}$ surface with 
only one single sharp peak, which can be easily designed in the frequency domain. By utilizing the CAF expression in terms of time-domain signals, the complexity of the proposed algorithm can be kept at a relatively low level. Simulation results are also provided to demonstrate the superior accuracy of this approach in frequency-selective fading channels and its robustness to RTO.

Notation: The superscript ${ }^{T}$ and ${ }^{*}$ denote transpose and complex conjugate, respectively; $\|\cdot\|$ denotes Euclidean norm; $\mathbf{C}(m,:)$ and $\mathbf{C}(:, n)$ denote the $m^{\text {th }}$ row and $n^{\text {th }}$ column of matrix $\mathbf{C}$, respectively; $\delta(\cdot)$ is the Dirac delta function; $\mathcal{N}_{\mathcal{C}}\left(\mu, \sigma^{2}\right)$ is a complex normal distribution with mean $\mu$ and variance $\sigma^{2}$; and $\chi^{2}(k)$ is a chi-square distribution with $k$ degrees of freedom.

\section{SySTEM MODEL}

Consider a discrete-time baseband OFDM system with the lengths of the IFFT/FFT and the CP equal to $N$ and $N_{g}$, respectively. $x(n)(0 \leq n \leq N-1)$ is the local training sequence for IFO estimation, which is generated as

$$
x(n)=\frac{1}{N} \sum_{k=0}^{N-1} X(k) e^{j 2 \pi k n / N}
$$

where $X(k)(0 \leq k \leq N-1)$ is the corresponding frequency-domain training sequence. Assuming that the length of the discrete-time channel impulse response (CIR) is $L$ and the channel is block stationary, we denote $\mathbf{h}=[h(0), h(1), \cdots, h(L-1)]^{T}$ and $H(k)=$ $\sum_{l=0}^{L-1} h(l) e^{-j 2 \pi l k / N}$ as the discrete-time CIR and the channel frequency response, respectively.

The time-domain training sequence is transmitted over a channel with frequency offset and corrupted by Gaussian noise. Assume that the RTO of $v$ samples is within the ISIfree region of the $\mathrm{CP}$, i.e., $v \in\left\{0,1, \ldots, N_{g}-L\right\}$, which means it will only introduce a linear phase rotation in each subcarrier. Then, the received time-domain training block can be expressed as

$$
y(n)=\sum_{l=0}^{L-1} h(l) x(n-v-l) e^{j\left(2 \pi \varepsilon(n-v) / N+\theta_{0}\right)}+w(n)
$$

where $w(n)$ is the time-domain complex AWGN; $\varepsilon$ and $\theta_{0}$ are the normalized IFO and initial phase, respectively. Since IFO is the focus here, we assume that the FFO has been perfectly estimated and compensated. After CP removal, the FFT output of the received training block is

$$
Y(k)=e^{j\left(\theta_{0}-2 \pi v k / N\right)} H(k-\varepsilon) X(k-\varepsilon)+W(k)
$$

where $W(k)$ represents the frequency-domain complex AWGN with zero mean and variance $\sigma_{\mathrm{n}}^{2}$.

\section{CAF BASED IFO Estimation}

Ambiguity functions (AF) were initially applied in target resolution and parameter estimation for radar systems [9]. Applications of AF can also be found in pulse design and optimization for OFDM [10]. Next, we introduce the AF and CAF and then apply them to the design of the training sequence and the establishment of an IFO estimation metric.

AF, a 2-D function of time delay and frequency offset (Doppler), is defined as the inner product of a signal and its time-delayed and frequency-shifted version [9]. Specifically, the AF of the local training sequence $x(n)$, denoted as $\mathbf{A}_{\mathbf{x x}}$, is

$$
A_{\mathbf{x x}}(\tau, \varpi)=\sum_{n=0}^{N-1} x(n) x^{*}(n-\tau) e^{-\frac{j 2 \pi \varpi n}{N}}
$$

where $\tau$ and $\varpi$ represent the time delay and frequency offset, respectively. As is well known, it is more convenient for OFDM systems to design the training sequence in the frequency domain. Hence, $\mathbf{A}_{\mathbf{x x}}$ can be derived equivalently using the frequency-domain signal $X(k)$, i.e.,

$$
A_{\mathbf{x x}}(\tau, \varpi)=\frac{1}{N} \sum_{k=0}^{N-1} X(k+\varpi) X^{*}(k) e^{\frac{j 2 \pi k \tau}{N}}
$$

Similarly, the CAF, denoted as $\mathbf{A}_{\mathbf{y x}}$, of the received training block $y(n)$ and the local training sequence $x(n)$ is

$$
A_{\mathbf{y x}}(\tau, \varpi)=\frac{1}{N} \sum_{k=0}^{N-1} Y(k+\varpi) X^{*}(k) \cdot e^{\frac{j 2 \pi k \tau}{N}}
$$

Moreover, the following variables are defined:

- $\left|\mathbf{A}_{\mathbf{x x}}\right|^{2}:$ AF surface, the set of $\left\{\left|A_{\mathbf{x x}}(\tau, \varpi)\right|^{2}\right\}$

- $\left|\mathbf{A}_{\mathbf{y x}}\right|^{2}:$ CAF surface, the set of $\left\{\left|A_{\mathbf{y x}}(\tau, \varpi)\right|^{2}\right\}$

- Doppler cut: an ambiguity function cut along the timedelay axis on the 2-D time-delay/frequency-offset plane

- $\boldsymbol{\Gamma}_{\varpi}=\mathbf{A}_{\mathbf{x x}}(:, \varpi)$ : the $\varpi^{\text {th }}$ AF Doppler cut

- $\boldsymbol{\Psi}_{\varpi}=\mathbf{A}_{\mathbf{y x}}(:, \varpi)$ : the $\varpi^{\text {th }}$ CAF Doppler cut

The maximum of the AF surface appears at $(0,0)$ [9], i.e.,

$$
\left|A_{\mathbf{x x}}(\tau, \varpi)\right|^{2} \leq\left|A_{\mathbf{x x}}(0,0)\right|^{2}
$$

It is well known that an AF is ideal if it is non-zero only at $(0,0)$, which is referred to as a thumbtack-type AF [11]; that is $\mathbf{A}_{\mathbf{x x}}(\tau, \varpi)=\delta(\tau) \cdot \delta(\varpi)$. Although this ideal function could yield superior target-resolution capabilities, it cannot be realized in practice [12], since there is no physically realizable signal that can produce $\delta(\tau) \cdot \delta(\varpi)$ according to the definition of the AF. Next, we will find a practical training sequence whose AF can approximate the ideal thumbtack-type AF. Then, by investigating the energy-distribution of the CAF, we will establish an energy-detection based IFO estimation metric to obtain a highly accurate IFO estimation in the presence of RTO.

\section{A. Energy Distribution of $A F$}

By investigating several commonly used training sequences in practical applications, a frequency-domain sequence (denoted by $X(k)$ ) with constant amplitude $(\lambda)$ and random phase is selected as the proposed training sequence for IFO estimation. Normally, $\{X(k)\}$ can be modeled as i.i.d. complex random variables (RV) with zero mean and variance $\lambda^{2}$. In order to verify the validity of the selected training sequence, the $\mathrm{AF}$ characteristics for $X(k)$ are analyzed as follows. By denoting $Z_{\tau, \varpi}(k) \triangleq X(k+\varpi) X^{*}(k) e^{j 2 \pi k \tau / N}$, we can rewrite (5) as 


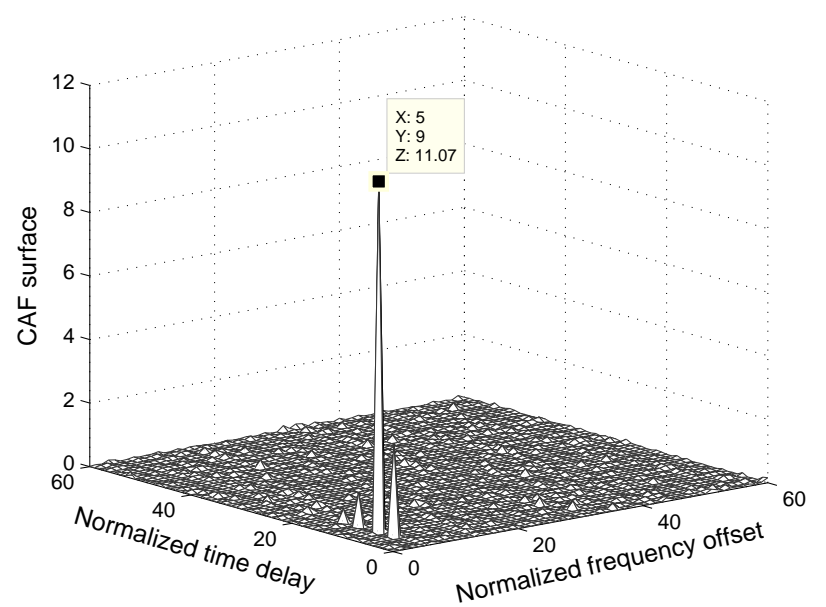

Fig. 1. CAF surface $(N=512, \lambda=2, \varepsilon=5, v=6, L=26$, $\left.\max \left\{|h(n)|^{2}\right\}=0.6918\right)$

$\Gamma_{\varpi}(\tau)=\frac{1}{N} \sum_{k=0}^{N-1} Z_{\tau, \varpi}(k)$. When $\varpi=0$, from (5), it is easy to show that the $0^{\text {th }}$ AF Doppler cut is

$$
\Gamma_{\varpi}(\tau)=\lambda^{2} \delta(\tau)
$$

For $\varpi \neq 0$, since $\{X(k)\}$ are i.i.d. RVs with zero mean and variance $\lambda^{2}$, it is easy to show that $\left\{Z_{\tau, \varpi}(k)\right\}$ are i.i.d. RVs with zero mean and variance $\lambda^{4}$. From the Central Limit Theorem and the fact that $\Gamma_{\varpi}(\tau)=\frac{1}{N} \sum_{k=0}^{N-1} Z_{\tau, \varpi}(k)$, we can deduce that $\left\{\Gamma_{\varpi}(\tau)\right\}$ are nearly identically distributed complex Gaussian RVs with zero mean and variance $\sigma_{\gamma}^{2}=$ $\lambda^{4} / N$, i.e.,

$$
\Gamma_{\varpi}(\tau) \sim \mathcal{N}_{\mathcal{C}}\left(0, \lambda^{4} / N\right), \text { for } \varpi \neq 0
$$

Then, from (8) and (9), the energy distribution of the AF of $X(k)$ can be obtained as follows:

1) From Parseval's theorem, $\left\|\boldsymbol{\Gamma}_{\varpi}\right\|^{2}=\lambda^{4}$ for any given $\varpi$ $(0 \leq \varpi \leq N-1)$, which implies that all AF Doppler cuts have the same energy.

2) Since $\left|\Gamma_{\varpi}(\tau)\right|^{2}=\lambda^{2} \delta(\tau)$ for $\varpi=0$, the energy of the $0^{\text {th }} \mathrm{AF}$ Doppler cut is concentrated at the $0^{\text {th }}$ time delay.

3) When $\varpi \neq 0$, from (9), we can show that $\left\{\left|\Gamma_{\varpi}(\tau)\right|^{2}\right\}$ are nearly identically distributed complex $\chi^{2}$-distributed $\mathrm{RVs}$ with degree of freedom 2. This implies that each element of the $\varpi^{\text {th }}(\varpi \neq 0)$ AF Doppler cut has an average energy of $\lambda^{4} / N$. Therefore, the total energy $\lambda^{4}$ of the $\varpi^{\text {th }}(\varpi \neq 0)$ AF Doppler cut is uniformly spread over all $N$ time delays in the statistical sense.

Based on the above properties, we see that the proposed training sequence has only a single sharp peak centered at $(0,0)$ on its AF surface. This verifies that the AF of a frequencydomain sequence with constant amplitude and random phase is a valid approximation of the ideal thumbtack-type AF.

\section{B. Energy Distribution of CAF}

Next, we derive the relationship between the CAF and the $\mathrm{AF}$ to determine the energy distribution of the CAF.
Substituting (3) into (6), the CAF in the presence of IFO and RTO is given by

$$
\begin{aligned}
A_{\mathbf{y x}}(\tau, \varpi)= & \frac{1}{N} \sum_{k=0}^{N-1}\left(e^{j\left(\theta_{0}-2 \pi v(k+\varpi) / N\right)} H(k+\varpi-\varepsilon)\right. \\
& \left.\times X(k+\varpi-\varepsilon) X^{*}(k) e^{\frac{j 2 \pi k \tau}{N}}+C(\tau, \varpi)\right)
\end{aligned}
$$

where $C(\tau, \varpi) \triangleq \frac{1}{N} \sum_{k=0}^{N-1} W(k+\varpi) X^{*}(k) e^{\frac{j 2 \pi k \tau}{N}}$ is the noise term. Since $\{W(k)\}$ are i.i.d. Gaussian RVs with distribution $\mathcal{N}_{\mathcal{C}}\left(0, \sigma_{\mathrm{n}}^{2}\right)$ and independent of $\{X(k)\}$, we can show that $\{C(\tau, \varpi)\}$ are identically distributed zero-mean complex Gaussian RVs with variance $\sigma_{\mathrm{g}}^{2}$. Since $\{X(k)\}$ have a constant amplitude $\lambda, \sigma_{\mathrm{g}}^{2}$ is given by $\sigma_{\mathrm{g}}^{2}=\lambda^{2} \sigma_{\mathrm{n}}^{2} / N$.

After some mathematical manipulations, the relationship between $\mathbf{A}_{\mathbf{y x}}$ and $\mathbf{A}_{\mathbf{x x}}$ is

$$
\begin{array}{r}
A_{\mathbf{y x}}(\tau, \varpi)=e^{j\left(\theta_{0}-2 \pi \varepsilon v / N\right)} \sum_{m=0}^{N-1}\left(e^{-j 2 \pi m(\varpi-\varepsilon) / N} h(m-v)\right. \\
\left.\times A_{\mathbf{x x}}(\tau-m, \varpi-\varepsilon)+C(\tau, \varpi)\right)
\end{array}
$$

Further, the relationship between $\Psi_{\varpi}$ and $\boldsymbol{\Gamma}_{\varpi}$ is

$$
\begin{aligned}
\Psi_{\varpi}(\tau)=e^{j\left(\theta_{0}-2 \pi \varepsilon v / N\right)} & \sum_{m=0}^{N-1}\left(e^{-j 2 \pi m(\varpi-\varepsilon) / N} h(m-v)\right. \\
& \left.\times \Gamma_{\varpi-\varepsilon}(\tau-m)+G_{\varpi}(\tau)\right)
\end{aligned}
$$

where $G_{\varpi}(\tau)$ is the $\tau^{\text {th }}(0 \leq \tau \leq N-1)$ element of $\mathbf{C}(:, \varpi)$.

From (12), we see that a CAF Doppler cut is a linear transformation of an AF Doppler cut corrupted by additive noise $G_{\varpi}(\tau)$. Based on this observation, the CAF is expected to have similar energy distribution properties as the AF; specifically,

1) Using (12) and the fact that $\left\|\boldsymbol{\Gamma}_{\varpi}\right\|^{2}=\lambda^{4}$, the energy of the $\varpi^{\text {th }}(0 \leq \varpi \leq N-1)$ CAF Doppler cut can be computed as

$$
\begin{aligned}
\left\|\Psi_{\varpi}\right\|^{2} & =\sum_{\tau=0}^{N-1}\left|\Psi_{\varpi}(\tau)\right|^{2} \approx\left\|\Gamma_{\varpi}\right\|^{2} \sum_{l=0}^{L-1}|h(l)|^{2}+N \sigma_{\mathrm{g}}^{2} \\
& =\lambda^{4} \sum_{l=0}^{L-1}|h(l)|^{2}\left(1+\eta^{-1}\right)
\end{aligned}
$$

where $\eta \triangleq \frac{\lambda^{2}}{\sigma_{n}^{2}} \sum_{l=0}^{L-1}|h(l)|^{2}$ is the signal-to-noise ratio (SNR). Hence, all CAF Doppler cuts have approximately the same energy. It also should be noted that the approximation in (13) will approach equality as $N$ and/or $\eta$ increases.

2) Using (12) and $\Gamma_{0}(\tau)=\lambda^{2} \delta(\tau)$, when the index of the CAF Doppler cut equals the true IFO $\varepsilon$ (i.e., $\varpi=\varepsilon$ ), we get

$$
\Psi_{\varpi}(\tau)=e^{j\left(\theta_{0}-2 \pi \varepsilon v / N\right)} \lambda^{2} h(\tau-v)+G_{\varpi}(\tau)
$$

Since $h(\tau)$ is defined over $\tau \in[0, L-1]$ and $v \in\left[0, N_{g}-L\right]$, we have

$$
\begin{aligned}
\sum_{\tau=v}^{v+L-1}\left|\Psi_{\varpi}(\tau)\right|^{2} & \approx \lambda^{4} \sum_{l=0}^{L-1}|h(l)|^{2}+L \sigma_{\mathrm{g}}^{2} \\
& =\lambda^{4} \sum_{l=0}^{L-1}|h(l)|^{2}\left(1+\frac{L}{N} \eta^{-1}\right)
\end{aligned}
$$

Similar to (13), the approximation in (15) will approach equality as $N$ and/or $\eta$ increases. Comparing (13) and (15), 
the normalized energy difference between them is given by

$$
\frac{\sum_{\tau=0}^{L-1}\left|\Psi_{\varpi}(\tau)\right|^{2}-\sum_{\tau=v}^{v+L-1}\left|\Psi_{\varpi}(\tau)\right|^{2}}{\sum_{\tau=0}^{L-1}\left|\Psi_{\varpi}(\tau)\right|^{2}} \approx \frac{N-L}{N} \cdot \frac{1}{1+\eta}
$$

Since $L$ is usually far less than $N$ for OFDM systems, the normalized energy difference in (16) can be approximated as $(1+\eta)^{-1}$. Therefore, the energy of the $\varepsilon^{\text {th }}$ CAF Doppler cut tends to concentrate on the $L$ time delays over $[v, v+L-1]$ with an increase in $\eta$. In this case, for the $\varepsilon^{\text {th }}$ CAF Doppler cut, the peaks of the CAF surface only appear within the interval $v \leq \tau \leq v+L-1$. Moreover, the peaks are approximately proportional to $|h(\tau-v)|^{2}$.

3) For $\varpi \neq \varepsilon$, using (12) and $\Gamma_{\varpi}(\tau) \sim \mathcal{N}_{\mathcal{C}}\left(0, \sigma_{\gamma}^{2}\right)$, we deduce that $\Psi_{\varpi}(\tau) \sim \mathcal{N}_{\mathcal{C}}\left(0, \sigma_{\psi}^{2}\right)$, where $\sigma_{\psi}^{2}=\sigma_{\mathrm{g}}^{2}+$ $\sigma_{\gamma}^{2} \sum_{l=0}^{L-1}|h(l)|^{2}=\frac{\lambda^{4}}{N} \sum_{l=0}^{L-1}|h(l)|^{2}\left(1+\eta^{-1}\right)$. Similar to AF Doppler cuts, when $\varpi \neq \varepsilon,\left\{\left|\Psi_{\varpi}(\tau)\right|^{2}\right\}$ are nearly identically distributed RVs which follow generalized chi-squared distribution. Hence, the total energy of the $\varpi^{\text {th }}(\varpi \neq \varepsilon)$ CAF Doppler cut is nearly uniformly spread over all $N$ time delays, in the statistical sense.

Therefore, we conclude that, for a frequency-domain training sequence with constant amplitude and random phase, the main sharp peaks only appear at the $L$ time delays over $[v, v+L-1]$ of the CAF surface of the proposed training sequence when $\varpi=\varepsilon$. CAF surfaces with ISI-free RTO are shown in Fig. 1.

\section{CAF Based IFO Estimation}

From (14) and the fact that $G_{\varpi}(\tau) \sim \mathcal{N}_{\mathcal{C}}\left(0, \sigma_{\mathrm{g}}^{2}\right)$, we can see that for $\varpi=\varepsilon, \Psi_{\varepsilon}(\tau)$ and $h(\tau)$ are correlated only for $v \leq \tau \leq v+L-1$. Moreover, from (9) and (12) we can also show that $\Psi_{\varpi}(\tau)$ and $h(n)$ are uncorrelated at all time delays for $\varpi \neq \varepsilon$. In other words, on the 2-D time-delay/frequencyoffset plane, $A_{\mathbf{y x}}(\tau, \varpi)$ and $h(n)$ are correlated only in the region $\{(\tau, \varpi) \mid v \leq \tau \leq v+L-1 ; \varpi=\varepsilon\}$. Therefore, if $h(n)$ is available for IFO estimation, an estimate of the IFO can be obtained by computing the moving cross-correlations of $h(n)$ and every CAF Doppler cut, $\Psi_{\varpi}(\tau)$, and finding the maximal modulus. Moreover, from the facts that $0 \leq v \leq N_{g}-L$ and $-\varepsilon_{\max } \leq \varpi<\varepsilon_{\max }$, the searching area is limited to $\left\{(\tau, \varpi) \mid 0 \leq \tau \leq N_{g}-1 ;-\varepsilon_{\max } \leq \varpi<\varepsilon_{\max }\right\}$. Denote $\hat{\varepsilon}$ and $\hat{v}$ as the trail values of IFO and RTO, respectively. An IFO estimator based on peak-detection of moving crosscorrelations can be constructed as

$$
(\hat{\varepsilon}, \hat{v})=\underset{\substack{-\varepsilon_{\max } \leq \hat{\varepsilon}<\varepsilon_{\max } \\ 0 \leq \hat{v} \leq N_{g}-L}}{\arg \max }\left|\sum_{\tau=\hat{v}}^{\hat{v}+L-1} e^{j 2 \pi \hat{\varepsilon} \hat{v}} h^{*}(\tau-\hat{v}) \Psi_{\hat{\varepsilon}}(\tau)\right|
$$

However, (17) is hard to realize since a valid channel estimation cannot be obtained before the IFO is estimated and compensated. Nevertheless, using (14), we can establish an estimate for $h(n)$ as

$$
\hat{h}(n)=e^{j 2 \pi \hat{\varepsilon} \hat{v} / N} \Psi_{\hat{\varepsilon}}(n+\hat{v}) / \lambda^{2}
$$

where $0 \leq n \leq L-1$ (the initial phase $\theta_{0}$ is absorbed into the channel estimation). This means that, for the proposed training sequence, a given $(\hat{\varepsilon}, \hat{v})$ corresponds to an estimate of the CIR $\hat{h}(n)$. Substituting (18) into (17), a CAF-based IFO estimation metric can be obtained as

$$
M(\hat{\varepsilon}, \hat{v})=\sum_{\tau=\hat{v}}^{\hat{v}+L-1}\left|\Psi_{\hat{\varepsilon}}(\tau)\right|^{2}
$$

Then, the resulting IFO estimation can be constructed as

$$
(\hat{\varepsilon}, \hat{v})=\sum_{-\varepsilon_{\max } \leq \hat{\varepsilon}<\varepsilon_{\max }, 0 \leq \hat{v} \leq N_{g}-L} \sum_{\tau=\hat{v}}^{\arg \max }\left|\Psi_{\hat{\varepsilon}}(\tau)\right|^{2}
$$

Note that $M(\hat{\varepsilon}, \hat{v})$ is just the energy of the $\hat{\varepsilon}^{\text {th }}$ CAF Doppler cut over $[\hat{v}, \hat{v}+L-1]$. From the energy distribution of the CAF in Sec. III-B, we can deduce that, in the high-SNR region, the maximum value of the energy in (19) will be obtained when $\hat{\varepsilon}=\varepsilon$ and $\hat{v}=v$. Moreover, since the domain of definition of $\varpi$ in CAF is $[-N / 2, N / 2), \varepsilon_{\max }$ can be up to $N / 2$; this means the proposed algorithm has full-range IFO estimation capability.

Next, we analyze the performance of the proposed IFO estimation from the AF and CAF energy distributions. For the proposed training sequence, an increase in $N$ will result in a sharper main peak around $(0,0)$ on its AF surface. In turn, for a given SNR $\eta$, a larger energy difference between the $\varpi^{\text {th }}$ $(\varpi=\varepsilon)$ CAF Doppler cut and any other CAF Doppler cut $(\varpi \neq \varepsilon)$ over $\tau \in[v, v+L-1]$ is consequently provided. On the other hand, it is obvious that an increase in $\eta$ will make the energy difference more distinct. Hence, the accuracy of the IFO estimation will be improved with an increase in $N$ and/or $\eta$. This can be alternatively explained from the aspect of the probability of incorrect IFO estimation. From the characteristics of the CAF we can show that, for an incorrect $\hat{\varepsilon}, M(\hat{\varepsilon}, \hat{v})$ in (19) has a generalized chi-squared distribution with mean $L \sigma_{\psi}^{2}$ and variance $L \sigma_{\psi}^{4}$, where $\sigma_{\psi}^{2}=\frac{1}{N}\left(\lambda^{2} \sigma_{n}^{2}+\right.$ $\left.\lambda^{4} \sum_{l=0}^{L-1}|h(l)|^{2}\right)=\frac{\lambda^{4}}{N} \sum_{l=0}^{L-1}|h(l)|^{2}\left(1+\eta^{-1}\right)$. Hence, for a given CIR length $L$, an increase in $N$ and/or $\eta$ will result in a decrease in both the expected value and variance. As a result, the probability of incorrect IFO estimation is reduced.

Compared with conventional approaches using two differentially encoded training sequences [5]-[6], the training overhead of the proposed algorithms is reduced since only one training sequence is required. In contrast to the DOFDM approaches [7], the restriction to an identical frequency response across adjacent subcarriers is relaxed. Therefore, the proposed algorithm is more robust to the frequency-selectivity caused by multipath channels. Compared with the ML-based IFO estimation method in [8], the proposed IFO estimator is more robust to the RTO.

\section{Equivalent Estimation Metric with Low Complexity}

From the above analysis we can see that, in order to obtain a full acquisition range IFO estimation, $N$ Doppler cuts of the CAF are involved in computing (19). From the definition in (6), the CAF can be obtained by $N$ IFFT operations. However, 


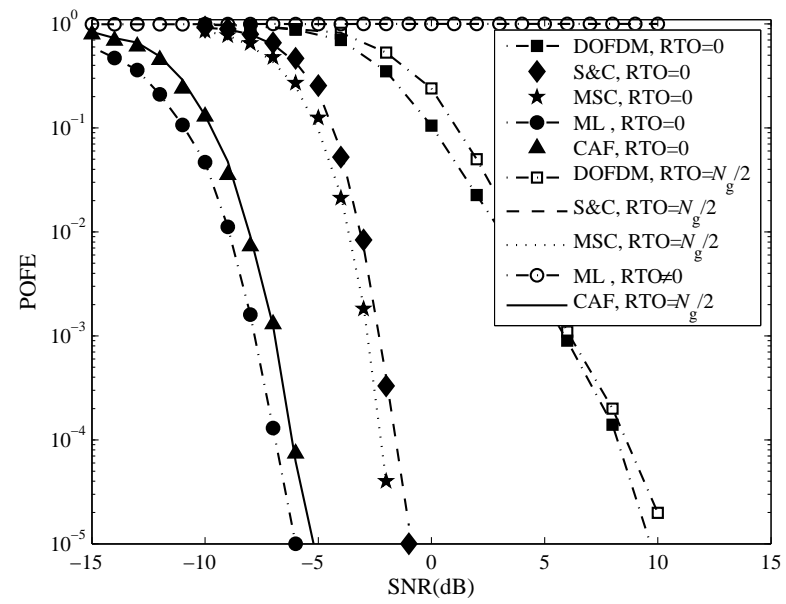

Fig. 2. Performance comparison for AWGN channels $(N=128)$

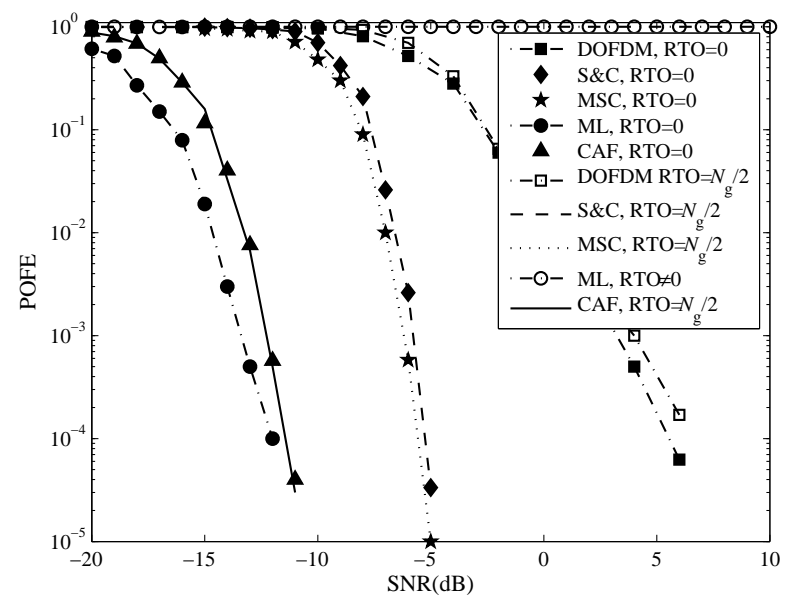

Fig. 3. Performance comparison for AWGN channels $(N=512)$

we can alternatively represent the CAF in terms of timedomain signals as

$$
A_{\mathbf{y x}}(\tau, \varpi)=\sum_{n=0}^{N-1} y(n) x^{*}(n-\tau) e^{-\frac{j 2 \pi \varpi n}{N}}
$$

where $\tau \in\left[0, N_{g}-1\right]$ and $\varpi \in[-N / 2, N / 2)$ represent the time delay and frequency offset, respectively. Obviously, in (21), only $L$ FFT operations are needed. By substituting (21) into (20), a low-complexity metric for the IFO estimation is given by

$$
(\hat{\varepsilon}, \hat{v})=\underset{\substack{-\varepsilon_{\max } \leq \hat{\varepsilon}<\max \\ 0 \leq \hat{v} \leq N g-L}}{\arg \max } \sum_{\tau=\hat{v}}^{\hat{v}+L-1}\left|\sum_{n=0}^{N-1} y(n) x^{*}(n-\hat{v}) e^{-\frac{j 2 \pi \hat{n} n}{N}}\right|^{2}
$$

It should be noted that, although (21) has a different expression from (6), the estimation performance will be unchanged since they have absolutely identical values.

\section{NumericAl RESUlTS}

The validity of the proposed IFO estimation algorithm is verified by computing the probability of failure estimation (POFE) defined as $\operatorname{Pr}\{\hat{\varepsilon} \neq \varepsilon\}$. Two typical IEEE 802.16e OFDMA parameter-sets, $\left(N=128 ; N_{g}=16\right)$ and $(N=512$; $\left.N_{g}=64\right)$, are adopted. The maximum normalized IFO is set as $\varepsilon_{\max }=N / 2$ (i.e., full acquisition range). The training symbols are randomly chosen from a QPSK constellation. For each SNR, at least $10^{6}$ independent trials are performed in which the IFOs are randomly generated within $[-N / 2, N / 2)$ and the RTOs are randomly generated within the ISI-free range. Specifically, the proposed algorithm is compared with four peer-algorithms: S\&C [5], modified S\&C (MSC) [6], DOFDM [7] and ML-based [8]. We simulated the ML algorithm using (15) in [8], and the RTO is incorporated in the received signal. For a fair comparison, we choose $J=1$ and $Q=1$, where $J$ and $Q$ represent the number of training sequences and identical segments, respectively.

Figs. 2-3 show the performance of different IFO estimators with and without RTO over AWGN channels. From the simulation results we can see that whatever the RTO is, the proposed CAF algorithm is far superior to the $\mathrm{S} \& \mathrm{C}$, MSC and DOFDM approaches. Although, in the case of perfect timing synchronization (i.e., $v=0$ ), the proposed algorithm is slightly inferior to the ML-based approach. The latter approach will fail to estimate the IFO as long as the RTO is not equal to zero. Compared with the ML-based algorithm, the other three estimators show good robustness to the RTO. Another observation from Figs. 2-3 is that an increase in the length of the FFT $(N)$ results in a performance improvement for all candidates. The reason for the performance improvement of CAF-based IFO estimation was analyzed in Sec. III-C.

The performance of five types of estimators over frequencyselective fading channels is shown in Figs. 4-6. From the parameters used, we can deduce that the maximal ISI-free RTOs are $16-6=10\left(N_{g}=16, L=6\right), 64-26=38$ $\left(N_{g}=64, L=26\right)$, and $64-51=13\left(N_{g}=64, L=51\right)$, respectively. Simulation results show that, as in Figs. 4-6, the CAF-based algorithm is significantly better than S\&C, MSC and DOFDM for all values of RTO. Moreover, these three approaches have good robustness to the RTO. Although, the ML-based algorithm slightly outperforms the CAF estimator when $\mathrm{RTO}=0$, it will deteriorate with an increase in RTO. Another important observation is that the ML-based method is very sensitive to a large RTO. As long as the RTO is greater than $L$, it fails to estimate the IFO.

Finally, the computational complexities of the five IFO estimators with full IFO acquisition range are shown in Table I in terms of the numbers of real multiplications and additions, respectively. For Example 1, the parameters are chosen as $N=128, N_{g}=16$, and $L=6$. For Example 2, $N=512$, $N_{g}=64$, and $L=6$. From these numerical results, we see that the overall complexity of the proposed CAF algorithm is the lowest.

\section{CONClusions}

In this paper, the problem of IFO estimation for OFDM systems with RTO and frequency-selective fading has been 
TABLE I

COMPLEXITY COMPARISON

\begin{tabular}{|c|ccc|ccc|}
\hline Algorithm & Real Multiplications & Example 1 & Example 2 & Real Additions & Example 1 & Example 2 \\
\hline S\&C & $N(4 N+2)$ & 65792 & 1049600 & $N(4 N-1)$ & 65408 & 1048064 \\
MSC & $4 N^{2}$ & 65536 & 1048576 & $N(4 N-2)$ & 65280 & 1047552 \\
DOFDM & $N(4 N+2)$ & 65792 & 1049600 & $N(4 N-1)$ & 65408 & 1048064 \\
ML & $4 N^{2}(L-1)-2 N L$ & 326144 & 5236736 & $2 N^{2}(2 L-1)-2 N L$ & 358912 & 5761024 \\
CAF & $2 N N_{g}\left(1+\log _{2} N\right)$ & 32768 & 655360 & $N N_{g}\left(1+2 \log _{2} N\right)-L N_{g}\left(N_{g}-L-1\right)$ & 31776 & 645248 \\
\hline
\end{tabular}

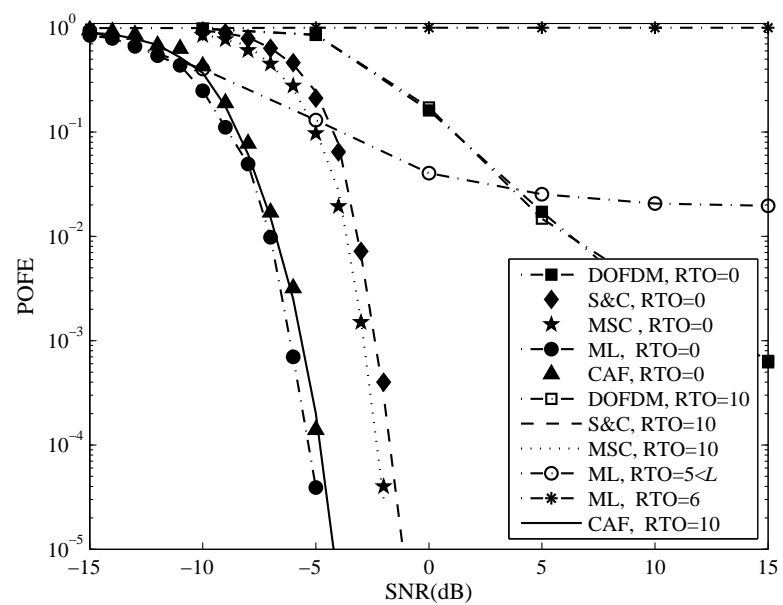

Fig. 4. Performance comparison for COST207 RA channels $(N=128$, $L=6)$

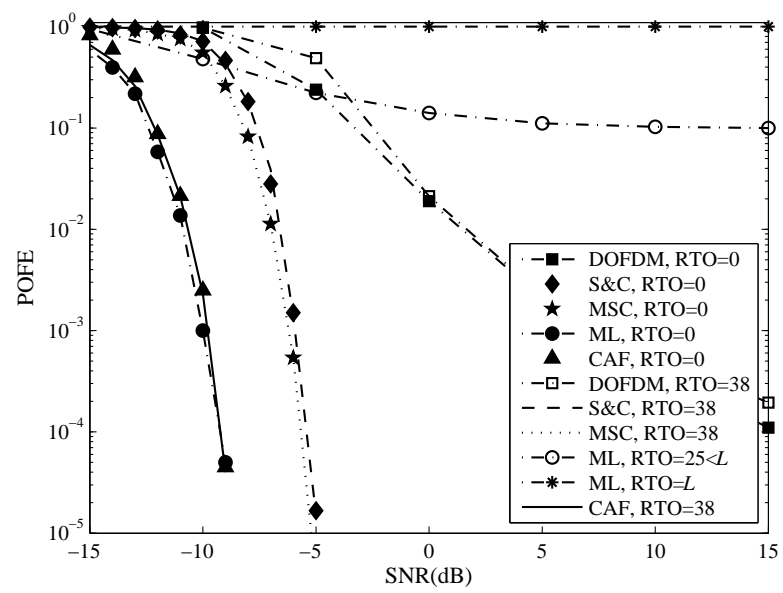

Fig. 5. Performance comparison for ITU VA channels $(N=512, L=26)$

considered. By analyzing the energy distribution of the AF, a constant-amplitude, random-phase sequence in the frequency domain which has only one single sharp peak on its AF surface is adopted as the training sequence. Moreover, by deriving the energy distribution of the CAF, an energy-detection based metric is established to obtain a highly accurate and full acquisition range estimation of the IFO in the presence of RTO. To guarantee low-level complexity, an equivalent CAF expression in terms of time-domain signals is utilized to reduce the number of FFT operations. Simulation results verify the validity and the robustness of the proposed algorithm.

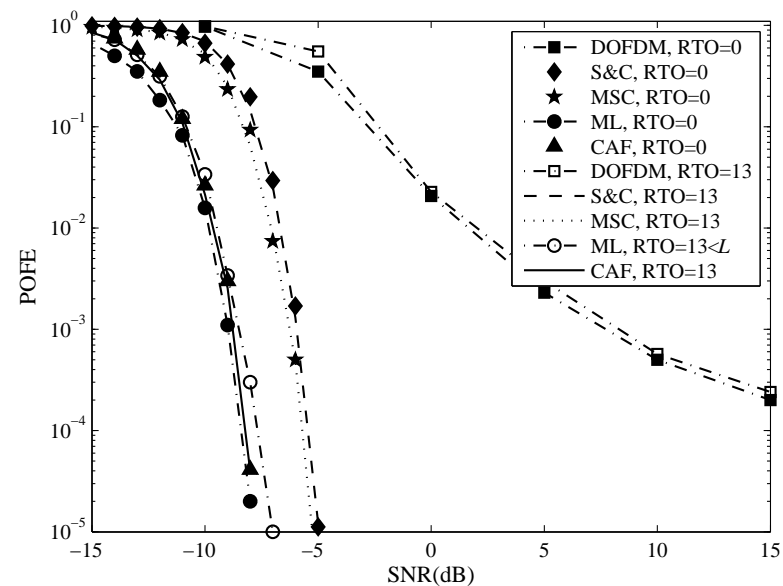

Fig. 6. Performance comparison for COST207 TU channels $(N=512$, $L=51$ )

\section{REFERENCES}

[1] L. Hanzo, Y. Akhtman, L. Wang, and M. Jiang, MIMO-OFDM for LTE WiFi and WiMAX: Coherent versus Non-coherent and Cooperative Turbo Transceivers. Wiley-IEEE Press, 2010.

[2] A. Al-Dweik, A. Hazmi, S. Younis, B. Sharif, and C. Tsimenidis, "Carrier frequency offset estimation for OFDM systems over mobile radio channels," IEEE Trans. Veh. Technol., vol. 59, no. 2, pp. $974-$ 979, Feb. 2010.

[3] H. Minn, V. Bhargava, and K. Letaief, "A robust timing and frequency synchronization for OFDM systems," IEEE Trans. Wireless Commun., vol. 2, no. 4, pp. 822-839, July 2003.

[4] X. Cai, Y.-C. Wu, H. Lin, and K. Yamashita, "Estimation and compensation of CFO and I/Q imbalance in OFDM systems under timing ambiguity," IEEE Trans. Veh. Technol., vol. 60, no. 3, pp. $1200-1205$, Mar. 2011.

[5] T. Schmidl and D. Cox, "Robust frequency and timing synchronization for OFDM," IEEE Trans. Commun., vol. 45, no. 12, pp. 1613-1621, Dec. 1997.

[6] C. Chen and J. Li, "Maximum likelihood method for integer frequency offset estimation of OFDM systems," Electron. Lett., vol. 40, no. 13, pp. 813-814, June 2004.

[7] Z. Liu, B. Weng, and Q. Zhu, "Frequency offset estimation for differential OFDM," IEEE Trans. Wireless Commun., vol. 4, no. 4, pp. 1737 -1748 , July 2005.

[8] M. Morelli and M. Moretti, "Integer frequency offset recovery in OFDM transmissions over selective channels," IEEE Trans. Wireless Commun., vol. 7, no. 12, pp. 5220-5226, Dec. 2008.

[9] S. Stein, "Algorithms for ambiguity function processing," IEEE Trans. Acoust., Speech, Signal Process., vol. 29, no. 3, pp. 588-599, June 1981.

[10] K. Liu, T. Kadous, and A. Sayeed, "Orthogonal time-frequency signaling over doubly dispersive channels," IEEE Trans. Inf. Theory, vol. 50, no. 11, pp. 2583-2603, Nov. 2004.

[11] M. Hussain, "Principles of high-resolution radar based on nonsinusoidal waves. II. Generalized ambiguity function," IEEE Trans. Electromagn. Compat., vol. 31, no. 4, pp. $369-375$, nov 1989.

[12] M. Jankiraman, Design of Multi-Frequency CW Radars. Scitech Pub Inc, 2007. 